\title{
Vittime di reato e centri di aiuto: riflessioni e proposte operative
}

\author{
Sofia Reatti
}

\begin{abstract}
Riassunto
Nel presente articolo vengono presentati i principali risultati emersi da uno studio empirico condotto nel 2014 presso il Centro per le vittime di reato e calamità di Casalecchio di Reno (BO). Scopo della ricerca, condotta per mezzo di una metodologia integrata di tipo qualitativo e quantitativo, è stato quello di studiare la storia di vita del Centro, ipotizzando anche la sua idoneità ad incrementare il senso di sicurezza percepita sul territorio. A tal fine la ricerca è stata sviluppata in due direzioni: da una parte ci si è avvalsi di una metodologia di tipo qualitativo per raccogliere informazioni in merito alla storia di vita del Centro, dall'altra, attraverso un approccio quantitativo, è stato somministrato un questionario al fine di sondare se e in quale misura la struttura sia conosciuta nel territorio, in che modo questa venga percepita in termini di utilità e quanto la sua presenza incida sul senso di sicurezza percepita dai cittadini.
\end{abstract}

\section{Résumé}

Dans cet article, l'auteur présente les principaux résultats d'une recherche menée en 2014 auprès du Centre d'aide aux victimes de crimes et de catastrophes (Centro per le vittime di reato e calamitä), de Casalecchio di Reno (BO - Italie), s'appuyant sur une méthodologie intégrée, basée sur des instruments qualitatifs et quantitatifs.

Le but de cette recherche était d'étudier l'histoire du centre, y compris en considérant son aptitude à renforcer le sentiment de sécurité des citoyens. En ce sens, la recherche a été divisée en deux volets : d'une part, grâce à une méthodologie qualitative, l'auteur a collecté des informations sur l'histoire du centre; d'autre part, sur le plan quantitatif, un questionnaire a été soumis afin de vérifier si et dans quelle mesure le centre est connu sur son territoire, la manière dont il est perçu par les citoyens, et son impact sur le sentiment de sécurité de la population.

\section{Abstract}

In this article the author presents the main results of a research carried out in 2014 at the Centre for crime and natural disaster victims (Centro per le vittime di reato e calamita) in Casalecchio di Reno (BO - Italy) using an integrated methodology based on qualitative and quantitative tools.

The aim of this research was to study the centre's history, even assuming its aptness to increase people's feeling of security. In this sense, the research was divided into two parts: on the one hand, the author worked with a qualitative methodology in order to gather information about the centre's history; on the other hand, from a quantitative point of view, a questionnaire was administered in order to verify whether and to what extent the Centre is known in their territory, how it is perceived by citizens: being useful or not, and what impact it has on people's feeling of security.

Key words: victim support; Italy; centre for crime and natural disaster victims; feeling of security; victimology.

\section{Introduzione.}

Sono numerose le associazioni che si sono costituite in seguito ad alcune delle tante stragi che hanno colpito il nostro Paese, la maggior parte di queste affronta la propria specifica realtà, altre hanno esteso il loro servizio di supporto a tutte le tipologie di vittime: è questo il caso del Centro per le vittime di reato e calamità di Casalecchio di Reno, nato in seguito alla strage che ha colpito l'istituto Salvemini nel 1990 (1).

Nonostante la presenza di centri di supporto alle vittime aspecifici sia ormai consolidata in buona parte del territorio europeo ed extra europeo, nel panorama italiano faticano ancora ad essere presi in considerazione. E' infatti noto come in Italia

\footnotetext{
- Dottoranda di ricerca in "Sociologia e ricerca sociale", XXXI ciclo, Dipartimento di Sociologia e Diritto dell’Economia, Unviersità di Bologna.
} 
azioni di sostegno alle vittime siano troppo spesso relegate a iniziative personali ed associative, senza un'adeguata copertura in termini di politiche sociali e di risorse anche in relazione ad una lacunosa normativa di riferimento (2). La realtà è nettamente differente in ambito europeo dove ad esempio i centri di victim support sono da anni istituzionalizzati e operano di concerto con le agenzie di controllo sociale presenti sul territorio, con positive ricadute anche in termini di coesione sociale e di vicinanza alle istituzioni locali.

Da questa consapevolezza è stata condotta una ricerca, sviluppata tramite una metodologia integrata di tipo qualitativo e quantitativo, che si propone di esaminare la storia di vita del Centro per le vittime e calamità di Casalecchio di Reno interrogandosi anche sul suo ruolo come soggetto che partecipa attivamente alla costruzione della sicurezza urbana nel territorio.

Si tratta di una struttura piuttosto diversa rispetto ad altre presenti sul territorio nazionale, diversità dovuta principalmente alla sua aspecificità che costituisce, di fatto, anche il suo maggior punto di forza. Gli operatori infatti si confrontano con molteplici problematiche: dalla violenza domestica alla truffa, da questioni di consumo a disagi sociali, tutte generano uno stato di disagio nella persona, anche quando questa non necessariamente è stata vittima di un reato. Centri di supporto alle vittime come questo sono ancora poco sviluppati e poco conosciuti in Italia, mentre sono una realtà ormai consolidata nel contesto internazionale.

\section{Metodologia della ricerca.}

E' stato svolto presso il Centro in esame uno studio sul campo, condotto attraverso il metodo dell'osservazione partecipante, che ha permesso di analizzarne la storia di vita mettendo a fuoco scopi e problematiche trattate, ruolo degli operatori e richieste degli utenti in termini di necessità e di motivazioni per le quali si rivolgono al Centro. Per fare ciò si è proceduto in due direzioni: da una parte le conoscenze acquisite sul campo, relativamente ai compiti della struttura ed ai suoi rapporti con le istituzioni sono state integrate con le informazioni ottenute attraverso interviste semistrutturate sottoposte ai professionisti che quotidianamente lavorano o collaborano con il Centro (coordinatore, operatore qualificato, psicologa, avvocato, assistente sociale) oltre che ai testimoni privilegiati del territorio in cui esso opera (Sindaco e comandante della Polizia Municipale); dall'altra, al fine di addivenire ad una comprensione più profonda della storia di vita della struttura, se ne è studiata l'evoluzione attraverso l'analisi secondaria dei dati relativi agli accessi nel periodo compreso tra il 2005 e il 2014 (3).

Si è trattato di attività necessarie al fine di giungere ad una comprensione il più possibile completa dell'attività svolta dagli operatori poiché "nel contesto della ricerca di tipo antropologico-etnografico (o studio sul campo, field-study) l'osservazione partecipante non rappresenta l'unico strumento del quale il ricercatore si serve. L'osservatore partecipante deve osservare, ascoltare e chiedere, e nel chiedere gli strumenti che adotta sono quelli dell'intervista. Nello stesso tempo deve documentarsi sui fatti che accadono e su quelli accaduti, esplorare il materiale documentario che già esiste sulla comunità oggetto di studio e quello che essa produce" (4). 
Si consideri poi che funzionale allo studio della storia di vita del Centro è stata l'attività di osservazione partecipante svolta, che ha permesso non solo di conoscere in dettaglio dinamiche interne, attività e problematiche trattate, ma anche di acquisire le basilari competenze nel sostegno alle vittime, coadiuvando gli interventi dei singoli operatori e partecipando attivamente alla risoluzione dei casi.

Nella seconda fase della ricerca ci si è interrogati circa la percezione del Centro da parte dei cittadini di Casalecchio di Reno, ipotizzando anche l'esistenza di un legame fra presenza della struttura sul territorio e sicurezza percepita dai cittadini.

Viviamo il quotidiano tra preoccupazioni globali e locali che sfuggono al nostro controllo, minano la nostra percezione di sicurezza e la nostra fiducia nelle istituzioni, siamo di fronte a paure globali cui sempre più rispondiamo a livello individuale, chiudendoci in noi stessi. Bauman introduce il concetto di società dell'incertezza, Beck di società del rischio, Putnam di indebolimento di capitale sociale e di "effetto tartaruga" e, d'accordo con lui, il sociologo statunitense Sennett elabora il concetto del "sé non collaborativo" con cui delinea il comportamento rinunciatario dei soggetti più restii a rivolgersi alle istituzioni e a collaborare fra loro per raggiungere uno scopo: “[...] sta emergendo una nuova tipologia caratteriale dai tratti specifici, la persona che non riesce a gestire forme complesse e impegnative di coinvolgimento sociale e quindi si chiude in se stessa" (ㅁ).

E' ormai noto come la percezione di sicurezza dei cittadini non dipenda solo dalla criminalità presente in un determinato territorio, ma sia anche il frutto di "una più ampia condizione esistenziale di incertezza legata ad ansie profonde e collettive. Queste ultime coinvolgono non solo l'aspetto della violazione della legalità (che va dagli atti di inciviltà alle fattispecie criminose), ma anche fattori di natura economico-sociale quali, ad esempio, i mutamenti nel mercato del lavoro, la stabilità del posto di lavoro, la crisi e il ridimensionamento del sistema di welfare, il mantenimento degli standard di vita, [...] dell'arrivo dei migranti, della carenza di servizi per le famiglie" (므). E' in questo contesto che assume particolare rilevanza il ruolo svolto dai centri di supporto alle vittime di reato, i quali adottano politiche di intervento volte a ripristinare il perduto senso di sé e a reintegrare il senso di responsabilità dell'individuo. Fra gli altri effetti delle attività svolte dagli operatori dei centri di victim support vi è poi quello di rinforzare e di ristabilire, laddove si sia perduto, il capitale sociale di un certo territorio, incentivando i legami e la coesione tra i cittadini e soprattutto il rapporto tra questi ultimi e le istituzioni. E' allora alla luce di queste considerazioni che si è ipotizzato come la presenza di un centro di supporto alle vittime di reato entro un determinato territorio possa efficacemente contribuire ad incrementare il senso di sicurezza percepita dei cittadini.

$\mathrm{Al}$ fine di ottenere dati empirici per poter sostenere ovvero confutare questa ipotesi di lavoro è stato predisposto e somministrato uno specifico questionario strutturato a cui hanno aderito 238 cittadini residenti o lavoratori attivi nel Comune di Casalecchio di Reno, interrogati su questioni concernenti criminalità, sicurezza (reale e percepita), eventuali esperienze 
vittimizzanti subite e conoscenza/percezione del Centro per le vittime.

\section{Il Centro per le vittime: uno spazio per superare l'offesa.}

L'edificio che prima ospitava l'istituto tecnico, distrutto dall'aereo in avaria, viene ricostruito grazie all'impegno e all'unione dei cittadini, delle associazioni di volontariato e delle istituzioni pubbliche del territorio con un'altra funzione rispetto a prima: rinasce come luogo di memoria e di aiuto. Nel 2001 viene così inaugurata a Casalecchio di Reno la Casa della Solidarietà, oggi sede del volontariato locale e della pubblica assistenza. Un modo per affrontare e superare il dolore, nella consapevolezza che "capire oltre ed altro rispetto a quanto si era già capito, anche se non è indolore, è una buona esperienza perché cambia le cose, apre nuove vie al fare [...]. $\mathrm{E}$ in questo modo, dopo appunto aver capito oltre ed altro rispetto a quello che si era capito in passato, si può forse proprio pensare di cambiare il passato: non $\mathrm{i}$ fatti in sé poiché ciò che è accaduto non è modificabile, ma i vissuti rispetto a quei fatti, almeno in parte è possibile cambiarli" (7).

E' questa presa di coscienza che sta alla base di tutta l'attività del Centro per le vittime di reato e calamità, nato nel 2005 proprio nei locali della Casa della Solidarietà.

Questa struttura può essere intesa come uno spazio di vita entro il quale persone che sono state vittime di qualsiasi tipo di reato, calamità o semplicemente di un'ingiustizia possono dare voce e riconoscimento ai propri diritti, ricevendo aiuto per districarsi fra $i$ difficoltosi aspetti giuridici, burocratici, amministrativi, oltre che emotivi che non di rado seguono l'evento dannoso, attraverso attività di sostegno, accompagnamento e consulenza. Uno spazio di tutela quindi che vuole essere un punto di riferimento per le parti sociali più deboli, ponendosi anche come interfaccia fra queste e le istituzioni. Che si tratti di semplici disagi o di più gravi situazioni di violenza, l'impegno degli operatori è quello di accogliere e cercare di risolvere le più disparate problematiche accomunate dal fatto di creare uno stato di disagio alla persona, che non necessariamente è vittima di reato, ed è precisamente questa aspecificità a costituire il carattere innovativo $\mathrm{e}$ distintivo di tale struttura rispetto alle altre presenti sul territorio.

Prima e fondamentale fase del percorso di aiuto è quella relativa all'accoglienza dell'utente: si tratta di un momento cruciale che, se gestito in modo non appropriato, può vanificare la stessa possibilità di attivazione del percorso. Altre attività in cui il Centro è quotidianamente impegnato riguardano il monitoraggio dei casi e dei relativi avanzamenti, l'aggiornamento dell'archivio, la gestione di uno specifico sportello a tutela dei consumatori nonché la valutazione, l'assegnazione e la gestione dei prestiti sull'onore. E' stata siglata una convenzione con le principali associazioni di consumatori per offrire aiuto anche a chi si trova di fronte a soprusi, inadempienze e forzature contrattuali, oggi sempre più frequenti nel campo delle utenze oltre che nel mercato assicurativo e finanziario. In molti casi i contenziosi sono di lieve entità e troppo spesso le vittime rinunciano ad avere giustizia poiché intrappolate nelle complesse maglie "burocratico-legali" dalle quali, senza un fattivo e concreto aiuto da parte di personale 
competente, difficilmente riuscirebbero a districarsi, rinunciando dunque a far valere i propri diritti.

L'aggravarsi della crisi economica ha portato poi gli operatori ad attivarsi anche nei confronti di tutte quelle persone che versano in uno stato di semi-indigenza che non permette loro di sostenere spese di mutui ipotecari, rate di locazione e forniture varie. E' stato quindi attivato lo strumento dei piccoli prestiti sull'onore per risolvere la momentanea situazione di disagio in cui versano questi soggetti, in attesa del recupero di una loro autonomia finanziaria.

Accanto a queste attività ordinarie, il Centro è periodicamente impegnato nell'organizzazione e nella gestione delle risorse economiche oltre che nei compiti di promozione e di sensibilizzazione, che hanno lo scopo di informare la cittadinanza in merito ai servizi offerti diffondendo la consapevolezza di situazioni vittimizzanti e fornendo indicazioni circa la possibilità di farvi fronte.

Le attività del Centro per le Vittime sono svolte esclusivamente da personale volontario, seppur coadiuvato da due psicologhe, un avvocato e due rappresentanti dell'associazione dei consumatori Adiconsum. Gli operatori presentano profili professionali eterogenei, alcuni sono privi di specifiche competenze e svolgono mansioni di carattere organizzativo, amministrativo e di segreteria, altri invece hanno una preparazione giuridica, psicologica o sociologica. A fronte della necessità di perseguire una certa omogeneità di comportamento e di acquisire gli strumenti idonei a rapportarsi in modo adeguato alle vittime nelle delicate fasi di accoglienza e di ascolto, è stata fornita una formazione di base in ambito criminologico e psicologico a tutti gli operatori volontari. Questo percorso formativo è stato affidato al CIRViS (Centro Interdisciplinare di Ricerca sulla Vittimologia e sulla Sicurezza dell'Università di Bologna), partner scientifico del progetto e all' Aspic, associazione che a Bologna si occupa di Counseling e Cultura.

Degna di nota è la significativa espansione territoriale e il conseguente incremento di utenza del Centro, la cui attività si è aperta dal 2010 a tutto il distretto nord di Casalecchio. Dai 25 casi trattati nel 2005, anno di inaugurazione, si è passati ai 500 del 2014, registrando ogni anno un incremento di utenza rispetto al precedente.

In merito alla prima fase della ricerca, dall'attività di analisi secondaria dei dati di accesso si evince che la stragrande maggioranza degli utenti $(81,8 \%)$ che si sono rivolti al Centro è di nazionalità italiana, solo l'11,9\% sono cittadini extra comunitari. Per quanto riguarda il genere, ogni anno gli accessi hanno riguardato in misura leggermente superiore le donne $(34,9 \%)$ rispetto agli uomini $(25,4 \%)$, un dato questo che si è mantenuto costante nel tempo e che rileva una sostanziale omogeneità fra i sessi. Relativamente all'età invece, i soggetti che si sono rivolti al Centro sono per la maggior parte $(31,3 \%)$ inclusi nella fascia di età compresa fra i 31 e i 45 anni e fra 46 e i 60 anni $(25,4 \%)$, seguiti da coloro che rientrano in quella dai 61 ai 75 anni (24\%). Giovani e anziani rappresentano invece le categorie residuali: gli accessi dei giovani fra i 18 e i 30 anni sono limitati al 5,9\% e il 6,1\% riguarda invece i soggetti che superano i 75 anni. Si registrano interessanti cambiamenti intervenuti nel tempo in merito alle problematiche presentate dagli utenti: alcune, come violenze domestiche e truffe, sono sempre 
state affrontate, altre invece si sono imposte all'attenzione degli operatori in tempi più recenti: il riferimento è in particolare alle problematiche derivanti dalla dipendenza dal gioco d'azzardo e a quelle legate alla crisi economica che, negli ultimi anni, hanno avuto un forte impatto sull'attività svolta.

In merito alle casistiche affrontate, l'opinione espressa dai testimoni privilegiati nel corso delle interviste trova una diretta conferma dall'analisi dei dati relativi agli accessi al Centro nel periodo compreso tra il 2005 ed il 2014: in questo arco di tempo i cosiddetti social problems, ovvero tutte quelle problematiche di disagio sociale riguardanti anche la sfera economica intra- familiare, sono quelli che maggiormente vengono affrontati nella quotidianità e che hanno subito una notevole impennata negli ultimi anni (grafico n. 1). Dall'analisi dei dati risulta infatti che i "disagi sociali e familiari" e le "questioni di consumo e prestazioni" sono progressivamente aumentate. Non si registrano invece significative variazioni nelle problematiche in funzione del genere se non per quanto riguarda i reati contro la persona, in questo caso infatti è nettamente più alta la percentuale di donne che hanno patito tali crimini $(14,1 \%)$ rispetto a quella degli uomini $(2,5 \%)$.

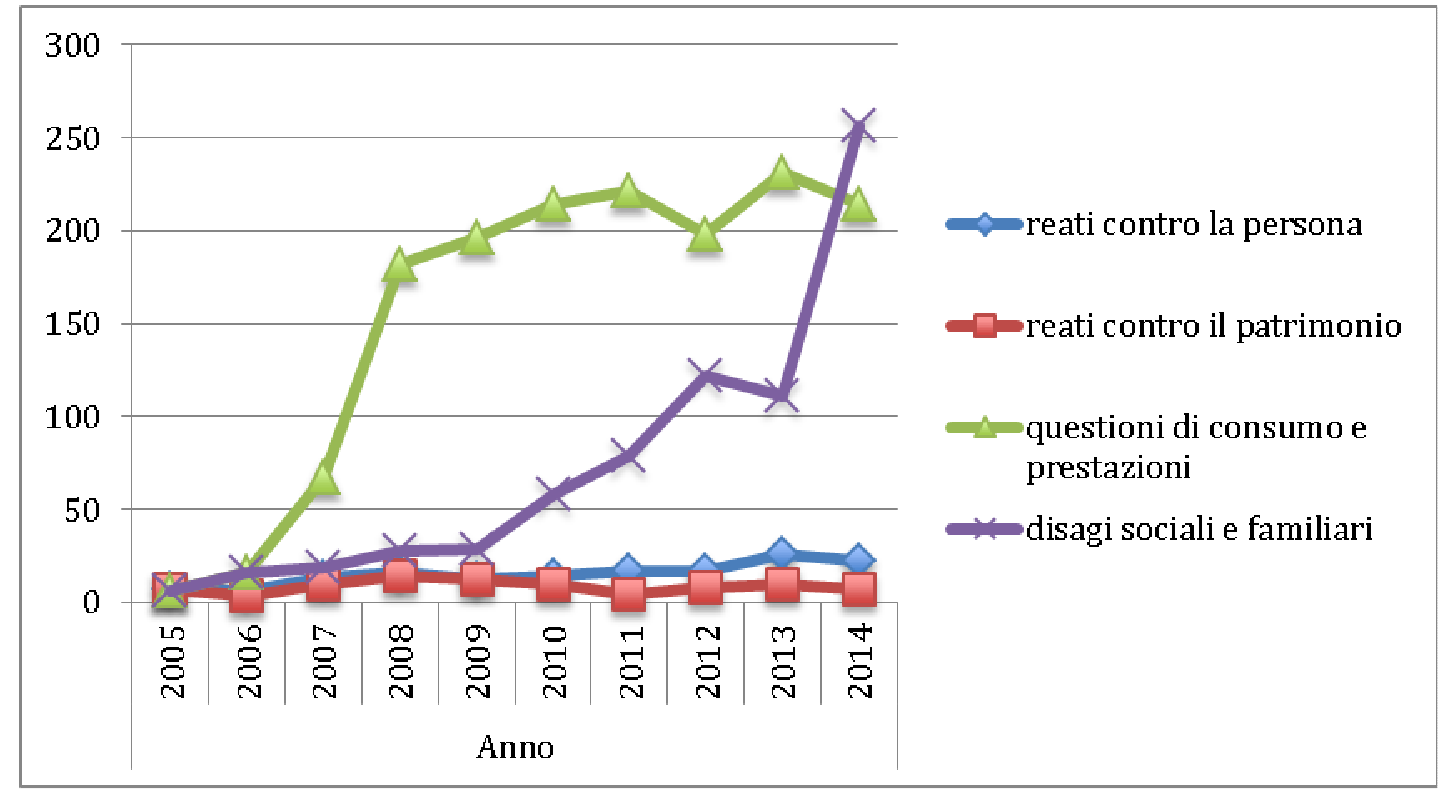

\section{Grafico n. 1 - Serie storica degli interventi effettuati dal Centro}

Dalle attività di osservazione partecipante e di analisi secondaria emergono due aspetti principali: un significativo incremento di utenza del Centro, ma anche alcune criticità legate essenzialmente alla precarietà del servizio. Dalle interviste effettuate e dall'analisi di secondo livello dei dati si evince non solo una notevole espansione territoriale del servizio, ma anche e soprattutto un sorprendente incremento di utenza: questi dati incoraggianti dimostrano che per i cittadini la struttura comincia ad essere un punto di riferimento e sembrano suggerire una maggiore consapevolezza circa la possibilità di ricevere aiuto. 
Si tratta però pur sempre di un'attività prettamente volontaria, salvo alcune collaborazioni di tipo professionale e, come afferma il coordinatore del Centro, è a rischio quella continuità di intervento e di presenza che fino ad oggi sono riusciti a mantenere. Inoltre gli operatori incontrano qualche difficoltà nella loro funzione di mediatori fra vittime e istituzioni, benché la presenza di un centro di supporto dovrebbe essere intesa come valore aggiunto all'interno di una data realtà territoriale.

\section{Il questionario.}

Si prende ora in considerazione la fase quantitativa dello studio: si è cercato di capire quale sia la percezione del Centro da parte della comunità di Casalecchio e soprattutto se esista un legame fra presenza della struttura sul territorio e sicurezza percepita dai cittadini. A tal fine è stato somministrato un questionario strutturato a un campione di soggetti, non precedentemente formato, residenti o lavoratori nel Comune di Casalecchio di Reno. Si è optato per una duplice modalità di somministrazione: $d e$ visu, nel territorio comunale anche in occasione di fiere ed eventi, ma anche on line, a distanza, coinvolgendo le principali associazioni del territorio presenti sui più comuni social network. Per quanto riguarda i primi, sono stati intervistati 128 soggetti fra commercianti e residenti, nei giorni dal 7 al 16 novembre 2014; i questionari on line invece sono stati inseriti su alcuni gruppi Facebook di cui si è avuta l'autorizzazione da parte degli amministratori e sono stati riproposti per tutto il mese di novembre 2014, raggiungendo 110 persone (ㅁ). Dei 238 questionari così ottenuti 22 non sono stati presi in considerazione perché incompleti e in fase di analisi si è pertanto lavorato su un totale di 216 questionari compilati correttamente. I dati ottenuti non sono generalizzabili all'intera popolazione del territorio di Casalecchio e il campione di riferimento non può e non vuole avere pretese di rappresentatività statistica.

Il questionario è composto di 29 domande con cui si vogliono cogliere atteggiamenti e quindi opinioni, sentimenti, giudizi e valutazioni, oltre che comportamenti dei cittadini in materia di sicurezza e di tutela della vittima (ㅁ).

Per agevolare il lavoro di analisi dei dati il questionario è stato suddiviso in due principali ambiti tematici, ciascuno dei quali è stato ulteriormente sviluppato in assi volti a specificare meglio i concetti $(\underline{10})$.

Il primo ambito tematico riguarda la sicurezza percepita dai cittadini e si sviluppa lungo i seguenti tre assi:

a. percezione della sicurezza: le domande hanno qui un intento meramente conoscitivo, in quanto si vogliono in via preliminare esplorare gli atteggiamenti dei cittadini in materia di sicurezza e quindi si raccolgono opinioni, valutazioni e giudizi in merito.

b. $\quad$ Esperienze di vittimizzazione subite: si ipotizza come l'essere stato vittima di un evento criminoso sia uno di quei fattori che più incidono sulla percezione di sicurezza del singolo, per questo si è ritenuto imprescindibile sondare questo aspetto.

c. Vicinanza alle istituzioni: dopo aver colto gli atteggiamenti si vogliono ottenere informazioni sui comportamenti adottati dalla vittima in seguito all'evento criminoso, in particolare per quanto riguarda l'eventuale denuncia e le persone e/o istituzioni cui si è chiesto aiuto. Avere informazioni sull'eventuale 
denuncia e sapere a quali persone/istituzioni preferisce rivolgersi chi ha patito un danno sono senz'altro elementi utili al fine di valutare il suo grado di vicinanza alle istituzioni.

Il secondo ambito tematico riguarda invece nello specifico la percezione del Centro Salvemini ed è stato anch'esso articolato lungo tre assi tematici:

a. Opinioni sulle necessità di una vittima e sull'utilità dei centri di supporto: si pongono qui domande relative agli atteggiamenti degli intervistati nei confronti della vittima e dei centri di supporto.

b. Conoscenza del Centro per le vittime "Salvemini": l'attenzione viene focalizzata sul Centro di Casalecchio per verificare se e quanto questo sia conosciuto e se l'intervistato vi si sia mai rivolto chiedendo poi di specificare, in caso

\begin{tabular}{|l|l|}
\hline Ambiti tematici & Assi tematici \\
\hline 1) Quale sicurezza a Casalecchio di Reno? & $\begin{array}{l}\text { a) percezione della sicurezza } \\
\text { b) esperienze di vittimizzazione subìte } \\
\text { c) vicinanza alle istituzioni }\end{array}$ \\
\hline 2) Il Centro Salvemini & $\begin{array}{l}\text { a) opinioni sulle necessità di una vittima e sull'utilità } \\
\text { dei centri di supporto } \\
\text { b) conoscenza del Centro per le vittime "Salvemini”" } \\
\text { c) valutazione del Centro }\end{array}$ \\
\hline Dati socio-anagrafici & $\begin{array}{l}\text { a) caratteristiche anagrafiche } \\
\text { b) caratteristiche sociali }\end{array}$ \\
\hline
\end{tabular}

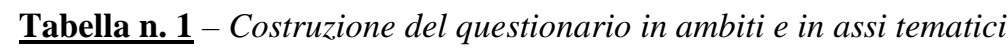

\section{Analisi dei risultati.}

\subsection{Chi sono gli intervistati.}

Dall'analisi dei dati socio-anagrafici si evince che il campione è composto per il 41,7\% (90) da uomini e per il 58,3\% (126) da donne. Nella quasi totalità dei casi i soggetti sono di nazionalità italiana, su 216 intervistati raggiunti infatti 213 sono italiani e solo 3 sono stranieri, si tratta tuttavia di un campione abbastanza eterogeneo sia per età sia per occupazione. Le di risposta affermativa, per quale motivo. Queste domande sono funzionali a sondare $i$ comportamenti dei soggetti, in particolare di coloro i quali hanno dichiarato di aver subito un reato, in funzione anche degli atteggiamenti che si sono rilevati verso i centri di supporto.

c. Valutazione del Centro: questo ambito è chiaramente riservato ai soli soggetti che hanno asserito di conoscere il Centro, chiamati ad esprimere un giudizio sullo stesso. 
frequentato le scuole dell'obbligo, di cui il 22,2\% (48) la scuola media inferiore. Meno rappresentati invece $i$ soggetti che hanno proseguito con gli studi universitari, che rappresentano solo il 12\% (26) del campione.

Per quanto riguarda l'occupazione svolta, la maggior parte degli intervistati, pari al 65,8\% (142), sono soggetti attivi dal punto di vista lavorativo, suddivisi tra lavoratori dipendenti 43,1\% (93) e lavoratori autonomi 22,7\% (49). Del 34,2\% (74) di inattivi, si tratta in buona misura di pensionati 19,4\% (42), seguiti da chi è in attesa di occupazione 6,5\% (14), studenti 6,\% (13) e casalinghe $2,3 \%$ (5). La somministrazione del questionario era subordinata alla conoscenza del territorio di Casalecchio di Reno e ne è stata pertanto richiesta la compilazione ai soli soggetti residenti o lavoratori nel Comune, infatti la stragrande maggioranza degli intervistati, pari all' $81,5 \%$ (176), risiede a Casalecchio e quasi la metà, il 46,3\% (100), vi lavora.

\subsection{La percezione della criminalità e della}

\section{sicurezza.}

Interrogati circa la percezione della criminalità nel comune di Casalecchio di Reno negli ultimi dieci anni, la stragrande maggioranza dei soggetti, pari al 70,4\% (152), afferma come questa sia aumentata, il 16,7\% (36) ritiene sia rimasta uguale e solo 9 persone sul totale $(4,2 \%)$ la percepiscono diminuita, mentre il restante $8,8 \%$ (19) non esprime un'opinione in merito.

E' interessante notare come nonostante la criminalità sia aumentata per buona parte degli intervistati, quasi la metà del campione, pari al $47,2 \%$ (102), dichiari comunque di sentirsi abbastanza sicuro. Dall'analisi dei dati e accorpando gli item "per niente sicuro"/"poco sicuro" e "sicuro"/molto sicuro" emerge tuttavia un quadro in cui la percezione di insicurezza è abbastanza elevata: infatti, è senza dubbio molto significativo che il 43,1\% (93) degli intervistati abbiano risposto di sentirsi poco e per niente sicuri contro la percentuale decisamente più esigua, 9,7\% (21), di coloro che si sentono sicuri e molto sicuri.

Da cosa dipende dunque la percezione di sicurezza degli intervistati? E' correlata a genere, età e titolo di studio? La variabile "genere" non sembra incidere in modo rilevante, le differenze fra uomo e donna infatti non sono significative se non forse per quanto riguarda la risposta "poco sicuro", in questo caso vediamo infatti un leggero aumento di insicurezza percepita da parte dei soggetti di genere femminile. Per quanto riguarda l'età, aggregando gli item "per niente sicuro" e "poco sicuro", si nota come la percezione di sicurezza diminuisca gradualmente con l'aumentare dell'età. Ad eccezione dei due soggetti che hanno 18 anni o meno che si sentono entrambi "per niente sicuri", la percezione di insicurezza è più bassa $(28,6 \%)$ nei giovani di età compresa fra i 19 e i 30 anni, ma aumenta considerevolmente al 43,1\% negli intervistati fra i 31 e i 45 anni e ancora di più per quelli di età fra i 46 e i 65 anni (49,2\%), rimanendo pressoché inalterata nelle persone di oltre 75 anni.

Un dato molto interessante si ottiene incrociando l'indicatore relativo alla "percezione di sicurezza" con il titolo di studio conseguito dagli intervistati: come si evince chiaramente dai dati riportati dalla tabella n. 1, esiste una diretta correlazione fra le due variabili, che si palesa in modo più evidente accorpando ancora una volta gli item "per niente sicuro" e "poco sicuro" e 
"molto sicuro" e "sicuro". La percezione di sicurezza è molto elevata nei soggetti con un basso livello di scolarizzazione (51,7\%), ma diminuisce al $43,2 \%$ in coloro che hanno proseguito gli studi superiori, mentre cala bruscamente al 23,1\% negli intervistati con un livello di istruzione universitaria. Fra questi ultimi si nota peraltro come nessuno affermi di sentirsi “per niente sicuro". Di contro, ovviamente, si riscontra come il 26,9\% degli intervistati che riferiscono una bassa percezione di insicurezza presentano un livello di istruzione universitaria a fronte di un 9,9\% con diploma di istruzione superiore e solamente $1{ }^{\prime} 1,7 \%$ con un livello di scolarizzazione basso. Si può quindi affermare che, per quanto riguarda il presente campione di intervistati, la percezione di insicurezza diminuisce considerevolmente con l'aumentare del titolo di studio. Tale correlazione viene efficacemente illustrata dal grafico riportato di seguito.

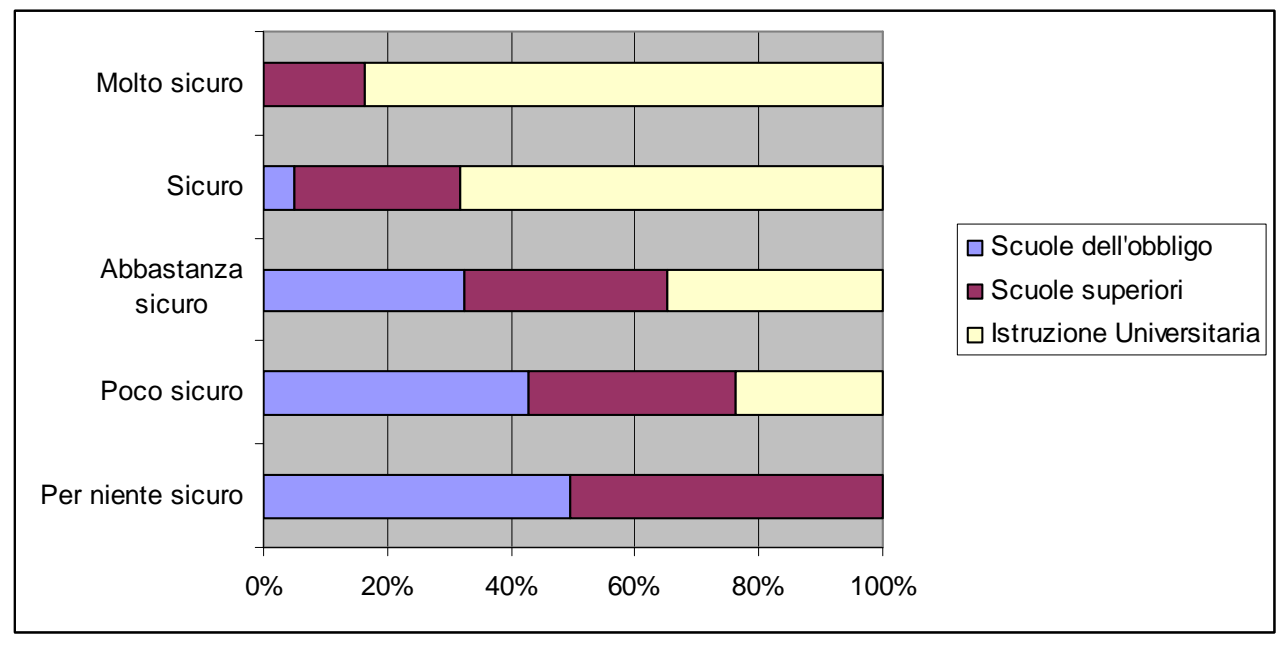

Grafico n. 2 - Percezione della sicurezza in funzione della scolarizzazione

La percezione della sicurezza è quindi trasversale al genere, ma correlata a età e titolo di studio. Si analizzano adesso le preoccupazioni degli intervistati: i dati indicano come i reati predatori, fra i quali furti (21,5\%), aggressioni/violenze $(13,5 \%)$ e rapine $(10,2 \%)$, siano i principali fattori ritenuti molto importanti nel concorrere a incrementare il sentimento di insicurezza. La presenza di immigrati si attesta al 15,1\% seguita dalle molestie sessuali $(9,2 \%)$. Si tratta di risposte prevedibili in funzione del particolare periodo in cui sono stati somministrati i questionari, contrassegnato da una situazione di generale allarme e di malcontento fra i cittadini a causa degli episodi di violenze e di furti che si sono verificati e che hanno probabilmente influenzato le risposte degli intervistati (11). Non destano invece particolare allarme prostituzione, spaccio di droga e atti vandalici.

Cosa invece fa sentire più sicuri i soggetti intervistati? La domanda "Quali di questi fattori Le infondono un maggior senso di sicurezza" propone una serie di item che si focalizzano su alcuni aspetti riguardanti sia politiche repressive ("presenza sul territorio di forze di polizia", "allontanamento di senza tetto, vagabondi, venditori ambulanti”) sia politiche preventive di 
tipo situazionale ("installazione di sistemi di videosorveglianza", “illuminazione stradale”, "potenziamento del trasporto pubblico in ore serali") e partecipative ("coinvolgimento dei cittadini in materia di sicurezza"), a questi si è aggiunto "presenza di centri di ascolto e di supporto alle vittime" al fine di verificare se e in quale misura un centro di supporto possa essere percepito come fattore che contribuisce a rinforzare il sentimento di sicurezza.

Da una prima lettura dei dati sembrerebbe che $\mathrm{i}$ fattori più importanti indicati siano la presenza di forze di polizia (36,7\%), illuminazione stradale $(18,3 \%)$ e “allontanamento di senza tetto e vagabondi". Ma un'analisi più puntuale si ottiene raggruppando ancora una volta gli item proposti: in questo modo infatti è evidente che gli intervistati accordano grande importanza alle politiche repressive per sentirsi più sicuri, 49,0\%, in particolare alla presenza delle forze dell'ordine, ma è rilevante anche il 12,3\% di intervistati che ha risposto "allontanamento di senza tetto e vagabondi". Anche le politiche di prevenzione situazionale sono comunque prese in considerazione da un significativo 34,9\% dei rispondenti, fra queste in particolare il potenziamento di illuminazione stradale è fortemente avvertito $(18,3 \%)$ come funzionale a sentirsi più sicuri, seguito dall'installazione di sistemi di videosorveglianza $(11,3 \%)$ e dal potenziamento del trasporto pubblico in ore serali (5,3\%). L'11,1\% dei cittadini ritiene molto importante essere coinvolto in materia di sicurezza mentre sono pochi $(5,0 \%)$ coloro i quali vedono nella presenza di centri di sostegno alle vittime un fattore che possa contribuire in modo importante a farli sentire più sicuri.
Dopo aver interrogato gli intervistati circa le loro opinioni in merito alla percezione della sicurezza, procedendo dal generale al particolare si chiede loro se siano stati vittima di reato negli ultimi dieci anni: oltre la metà, il 57,4\% (124), dei rispondenti dichiara di non essere stata colpita da alcun crimine. Il rimanente 42,6\% (92) viene invitato a specificare il tipo di reato di cui è rimasto vittima: la stragrande maggioranza di loro, pari al 73,4\%, dichiara di avere subito reati contro il patrimonio, fra questi in particolare il furto $43,0 \%$ (58), mentre il restante $26,6 \%$ è stato colpito da reati contro la persona.

Quali caratteristiche hanno le vittime e qual è la loro percezione della sicurezza? Si registrano differenze rispetto a chi invece non ha vissuto esperienze vittimizzanti? In primo luogo è interessante notare come vi sia una correlazione fra genere e vittimizzazione: come si evince dalla tabella n. 2 infatti, la percentuale di chi ha subito un reato è di 38,9\% per gli uomini e sale al $45,2 \%$ per le donne. Per quanto riguarda l'età, le percentuali di chi ha patito un crimine sono più elevate fra le persone che hanno un'età compresa fra i 31 e i 65 anni.

\begin{tabular}{|c|r|r|}
\hline \multirow{2}{*}{} & \multicolumn{2}{|c|}{ Genere } \\
\cline { 2 - 3 } & \multicolumn{1}{|c|}{$\mathbf{M}$} & \multicolumn{1}{c|}{$\mathbf{F}$} \\
\cline { 2 - 3 } Vittima & $\begin{array}{c}\text { \% casi } \\
\text { colonna }\end{array}$ & $\begin{array}{l}\text { \% } \\
\text { coloni } \\
\text { colona }\end{array}$ \\
\hline Sì & $38,9 \%$ & $45,2 \%$ \\
\hline No & $61,1 \%$ & $54,8 \%$ \\
\hline
\end{tabular}

Tabella 2 - Vittimizzazione in funzione del genere

Quale rapporto intercorre invece fra vittimizzazione e sicurezza percepita? Nonostante sia stato rilevato che "[...] il soggetto che ha subito un reato non rielabora automaticamente i propri meccanismi percettivi 
verso un atteggiamento di maggiore timore [...]" (12), nel caso del campione in esame e senza alcuna pretesa di generalizzazione, l'avere subito episodi vittimizzanti ha invece una diretta ricaduta sulla sicurezza percepita. Come riportato dalla tabella n. 3, sommando le risposte date a "per niente sicuro" e "poco sicuro", è evidente come la percentuale delle vittime di reato che affermano di sentirsi per niente o poco sicure superi la metà $(56,5 \%)$ e scenda notevolmente al 33,0\% per chi invece non è mai stato colpito dal crimine. Una possibile interpretazione di questo dato potrebbe essere che " $[. .$.$] il processo di vittimizzazione ha$ effetto pregiudizievole sul sentimento di appartenenza ad una comunità che si correla ad un intenso senso di abbandono e di messa in discussione dei valori culturali basilari” (므). E' vero che non sempre l'avere subito un reato si traduce in un maggiore senso di insicurezza, ma è altrettanto vero che la vittima, resa più vulnerabile dall'evento criminoso, può reagire ripiegandosi su sé stessa e, quando non supportata adeguatamente dalla rete di controllo formale ed informale, ciò può avere importanti ricadute in termini di sicurezza percepita.

\begin{tabular}{|l|r|r|}
\hline \multirow{2}{*}{} & \multicolumn{2}{|c|}{ Vittima } \\
\cline { 2 - 3 } & \multicolumn{1}{|c|}{ Si } & \multicolumn{1}{c|}{ No } \\
\cline { 2 - 3 } & $\begin{array}{c}\text { \% casi } \\
\text { colonna }\end{array}$ & $\begin{array}{c}\text { \% casi } \\
\text { colonna }\end{array}$ \\
\hline $\begin{array}{l}\text { Per niente } \\
\text { sicuro }\end{array}$ & $18,5 \%$ & $2,4 \%$ \\
Poco sicuro & $38,0 \%$ & $30,6 \%$ \\
& $38,0 \%$ & $54,0 \%$ \\
$\begin{array}{l}\text { Abbastanza } \\
\text { sicuro }\end{array}$ & $5,4 \%$ & $11,3 \%$ \\
Sicuro & $0,0 \%$ & $1,6 \%$ \\
Molto & & \\
sicuro & \\
\hline
\end{tabular}

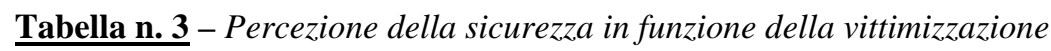




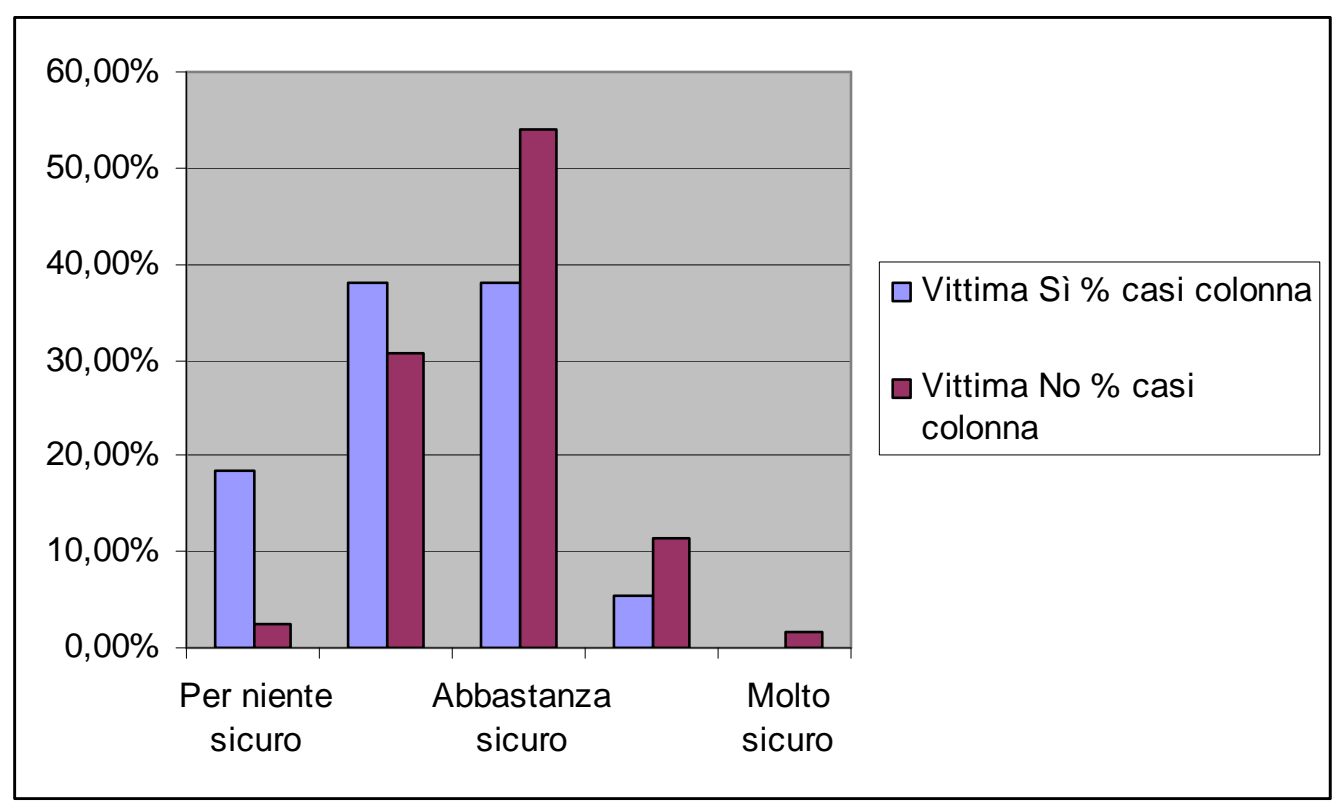

Graficon. 3 - Percezione della sicurezza in funzione della vittimizzazione

Dei soggetti che hanno patito un crimine, solo il $7,9 \%$ (17) non si è rivolto alle Forze dell'Ordine, mentre la maggior parte, il 34,7\% (75), dichiara di aver sporto denuncia: il 29,6\% (64) di loro non si è rivolto a nessun altro per ricevere aiuto, del rimanente 6\% (13) la maggior parte, 38,5\% (10), si è affidata a una persona cara, il 30,8\% (8) a un avvocato, il $15,4 \%$ (4) a uno psicologo, il $7,7 \%$ (2) ad associazioni di consumatori e centri di aiuto alle vittime.

Per quanto riguarda invece le vittime che non hanno denunciato l'offesa patita, oltre la metà $(60 \%)$ di loro ritiene molto importante la scarsa fiducia nell'operato delle forze dell'Ordine come motivo di non denuncia, seguito da "non volevo ricordare" $(42,9 \%)$.

A prescindere dall'aver sporto denuncia o meno, se questi soggetti dovessero in futuro rimanere ancora vittime di reato si rivolgerebbero nuovamente e in buona parte, $61,9 \%$ (78), alle forze dell'ordine, il 12,7\% (16) a un avvocato, il $7,9 \%$ (10) a una persona cara, il 6,3\% (8) a centri di aiuto, il 4,8\% (6) ad associazioni di consumatori, il 4,0\% (5) a uno psicologo e il $2,4 \%$ (3) a nessuno. Dall'analisi dei dati si evince come la stessa domanda posta a chi invece non ha subito alcun reato porta a risultati quasi analoghi: non si registrano infatti evidenti differenze nelle loro risposte rispetto a quelle fornite dalle vittime: anche in questo caso la maggior parte, il 54,9\% (123), degli intervistati si rivolgerebbe alle forze dell'ordine e, in seconda battuta, a un avvocato, 14,7\% (33).

Emerge complessivamente un quadro in cui le forze dell'ordine rimangono il punto di riferimento per $\mathrm{i}$ cittadini in difficoltà, ma si ritiene comunque significativa la percentuale di risposte date a "centri di aiuto", più elevata rispetto ad altri item.

\subsection{Il Centro "Salvemini”" visto dai cittadini.}

Vediamo ora in particolare quale visione hanno $i$ soggetti intervistati del Centro per le vittime di Casalecchio cercando anche di dare una lettura dei loro atteggiamenti e delle loro opinioni in 
merito alla funzione dei centri di sostegno e alle necessità di una vittima.

Qual è il parere degli intervistati in merito alle esigenze più urgenti di chi ha subito un reato o un'ingiustizia? La tabella n. 4 illustra che i primi tre fattori più importanti vengono ravvisati nel 27,3\% dei casi nell'ottenere giustizia, nel poter contare sulle istituzioni (22,6\%) e nel sentirsi protetto da minacce e ritorsioni (20,5\%), seguiti da essere ascoltato $(11,5 \%)$ e recuperare la normale quotidianità $(10,1 \%)$, mentre solo l' $8 \%$ invece indica quale fattore molto importante "ricevere aiuto e informazioni".

Cosa ne pensano invece gli intervistati in merito ai centri di supporto alle vittime? Oltre la metà del campione, pari al 68,5\% (148), ritiene siano utili a soddisfare le necessità di chi ha subito un reato. E' interessante a riguardo sottolineare come questo dato possa essere correlato ai risultati ottenuti da altre due ricerche presenti in letteratura che, anche se si sono avvalse di un campione sicuramente più ampio e rappresentativo rispetto a quello qui preso in considerazione, sono giunte a risultati analoghi. La prima indagine cui si fa riferimento, condotta allo scopo di delineare il profilo delle vittime di reato e di esplorarne il percorso evolutivo nella realtà emiliano-romagnola (14), prevede uno specifico quesito in merito a una maggiore diffusione dei servizi di aiuto alle vittime, a cui hanno risposto in modo favorevole il 92,7\% degli intervistati. L'altra ricerca, anch'essa volta ad analizzare la figura della vittima di reato, pone la stessa domanda a un campione di studenti dell'Università di Bologna, anche in questo caso la quasi totalità di loro $(96,2 \%)$ afferma di essere favorevole a una maggiore diffusione dei centri di supporto alle vittime (15).
Nelle ricerche appena menzionate le domande poste vertevano sulla diffusione dei centri di sostegno, in quella in esame invece si richiede un giudizio circa la loro utilità e la percentuale di soggetti che rispondono in senso affermativo, anche se elevata, non raggiunge la quasi totalità del campione come nelle altre indagini. E' inoltre interessante notare come la percezione di utilità dei centri di supporto sembri essere condizionata dall'essere stato o meno vittima di reato. Dalla lettura dei dati riportati dalla tabella n. 5 infatti si evince come le percentuali di risposte che indicano il Centro come utile siano più basse, $66,3 \%$, per chi è stato vittima e più alte, 70,2\% per chi non lo è stato, da ciò si potrebbe quindi dedurre che i centri sono ritenuti meno utili da chi ha subito un reato. Non incidono invece le variabili relative a genere ed età: le percentuali infatti sono distribuite in modo sostanzialmente uniforme senza differenze degne di nota. 


\begin{tabular}{|l|r|r|r|}
\hline & \multicolumn{2}{|c|}{ Risposte } & $\begin{array}{r}\text { Percentuale di } \\
\text { casi }\end{array}$ \\
\cline { 2 - 3 } & N & Percentuale & $23,2 \%$ \\
Essere ascoltato & 49 & $11,5 \%$ & $55,0 \%$ \\
Ottenere giustizia & 116 & $27,3 \%$ & $41,2 \%$ \\
Sentirsi protetto da minacce & 87 & $20,5 \%$ & \\
Ricevere aiuto e & 34 & $8,0 \%$ & $16,1 \%$ \\
informazioni & & & \\
Poter contare sulle istituzioni & 96 & $22,6 \%$ & $45,5 \%$ \\
Recuperare la normale & 43 & $10,1 \%$ & $20,4 \%$ \\
quotidianità & 425 & $100,0 \%$ & $201,4 \%$ \\
\hline
\end{tabular}

Tabella n. $\mathbf{4}$ - Necessità per le vittime (molto importante)

\begin{tabular}{|l|r|r|}
\hline \multirow{2}{*}{} & \multicolumn{2}{|c|}{ Vittima } \\
\cline { 2 - 3 } & \multicolumn{1}{|c|}{ Sì } & \multicolumn{1}{c|}{ No } \\
\cline { 2 - 3 } & \multicolumn{1}{c|}{ \% casi } & \% casi \\
colonna & colonna \\
\hline Sì & $66,3 \%$ & $70,2 \%$ \\
\hline No & $33,7 \%$ & $29,8 \%$ \\
\hline
\end{tabular}

Tabella n. 5 - Utilità centri di supporto in funzione della vittimizzazione

Soffermiamoci ora sul 31,5\% (68) di intervistati per i quali i centri non sono utili e vediamo per quale motivo. Ciò che emerge dalla lettura dei dati è che per la maggior parte di loro, 25\% (54), non risolvono davvero i problemi, mentre solo tre soggetti $(1,4 \%)$ ritengono che vi siano già altri servizi cui rivolgersi.

Anche in questo caso si può ravvisare un'analogia con i risultati della già menzionata ricerca sulla figura della vittima di reato (16), dove viene chiesto all'esigua percentuale di studenti contrari ad una maggiore diffusione dei centri di supporto di giustificare il motivo. Fra gli item proposti "sul territorio esistono già i servizi sociali", "sono solo una facciata per i politici" e "non risolvono davvero i problemi" sono gli ultimi due a ricevere il maggior numero di risposte.

Questi dati potrebbero essere letti come conseguenza di un diffuso sentimento di sfiducia verso le istituzioni in generale che può portare a tenere atteggiamenti improntati a diffidenza, scetticismo e quindi chiusura verso ciò che è nuovo e che non si conosce. Se a questo si aggiunge la carenza di quella "cultura vittimologica" che nel nostro Paese ancora fatica a nascere, può darsi che la diffusione e la percezione di utilità dei centri di sostegno alle vittime di reato sia avvertita come qualcosa di superfluo e di non prioritario.

A sostegno di questa lettura devono essere prese in considerazione le risposte date dagli intervistati all'item "altro": quattro persone $(1,9 \%)$ non ritengono utili questi servizi perché non li conoscono, altri rispondono "non mi sento coinvolto nella loro attività", "non tutelano nel tempo", "il buonismo dilagante non aiuta" e "giustizia e protezione dovrebbero essere compito delle forze dell'ordine".

La scelta stessa dell'item "altro", evitato invece nella maggior parte degli altri quesiti, è a parere di chi scrive un dato da ritenersi significativo in 
quanto denota la volontà di esprimersi ulteriormente sulla questione. Anche se si tratta di pochissimi intervistati, infatti, questi hanno comunque sentito l'esigenza di specificare meglio il motivo della loro risposta.

Infine, incrociando i motivi indicati per giustificare la non utilità dei centri di supporto con le variabili di genere e vittimizzazione non emergono differenze degne di nota in base al genere, più significative invece sono le risposte date in funzione dell'età: come si vede chiaramente dalla tabella n. 6 le percentuali delle risposte date a "non risolvono davvero i problemi" aumentano progressivamente con l'aumentare dell'età.

$\mathrm{Al}$ di là di queste, pur necessarie, considerazioni ciò che è importante e che merita di essere nuovamente sottolineato è che, sul totale del campione, la percentuale di coloro che ritengono utili i centri di supporto alle vittime è elevata e significativa. Questo è il dato principale, forse indicativo del fatto che qualcosa sta lentamente cambiando e segnale di una crescente attenzione e sensibilità verso le esigenze di una vittima.

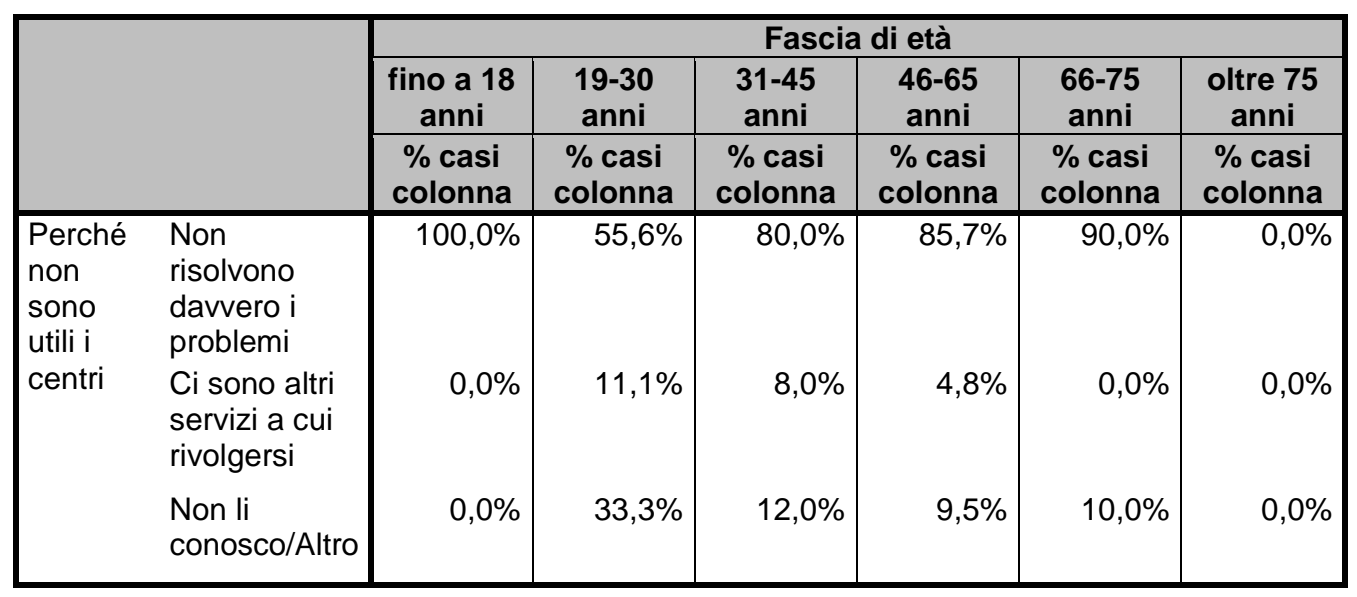

Tabella n. 6 - Motivazioni non utilità dei centri in funzione dell'età

I dati sulla conoscenza del Centro per le vittime di reato e calamità di Casalecchio sono forse $i$ più significativi della ricerca in esame: è infatti emblematico come a dieci anni dalla sua istituzione e nonostante $\mathrm{i}$ numerosi convegni di sensibilizzazione e promozione del servizio, questo sia conosciuto solo da un'esigua percentuale, pari al 38,4\% (83), dei soggetti intervistati e come solo il 5,6\% (12) di questi vi si sia rivolto per chiedere aiuto. Tra le persone che conoscono il Centro, ma non vi si sono mai rivolti, il 28,2\% (61) afferma di non averne mai avuto bisogno, non sapeva esattamente di cosa si occupasse il 3,2\% (7) e il 2,8\% (6) ha preferito affidarsi ad altri.

E' interessante notare come il Centro sia conosciuto in misura maggiore $43,5 \%$ dalle persone che sono state vittime e meno $(34,7 \%)$ da quelle che non lo sono state, questo può forse significare che le campagne di sensibilizzazione promosse dal Centro hanno raggiunto l'attenzione dei soggetti più vulnerabili.

Un dato che sembra confermare la diffidenza verso queste strutture è che delle 44 vittime che conoscono il Centro solo 9 vi si sono rivolte per chiedere aiuto. 
Circoscrivendo l'analisi agli intervistati che si sono rivolti al Centro, emerge come la maggior parte di loro lo abbia fatto a seguito di un reato: il 40,9\%, infatti ha subito reati contro il patrimonio (in particolare furto, rapina, scippo e truffa) e il $13,5 \%$ reati contro la persona (lesioni personali, minacce e mobbing); degli altri, il $22,7 \%$ (5) ha chiesto aiuto per risolvere difficoltà economiche e il $22,7 \%$ per controversie amministrative e per gestire $i$ rapporti con la pubblica amministrazione.

Quale idea ha del Centro per le vittime chi lo conosce? Si vuole verificare se il servizio offerto dalla struttura sia stato effettivamente d'aiuto all'utente: a parte l'8,3\% (18) degli intervistati che non vi si sono mai rivolti, è significativo che per il $3,3 \%$ lo sia stato poco o per niente, "abbastanza" per il 2,3\% e solo 2 persone $(0,9 \%)$ hanno risposto "molto". Nonostante questo dato, complessivamente la maggior parte dei soggetti, 19\% (41), che conosce il Centro per le vittime lo consiglierebbe a un amico in difficoltà. In ultima analisi, la presenza del Centro Salvemini non sembra incidere in modo significativo sul sentimento di sicurezza percepita del cittadino. Stando ai dati infatti buona parte degli intervistati, 21,3\% (46), che conosce il Centro dichiara che questo non influenza la propria percezione di sicurezza, anche se può ritenersi comunque significativo il $15,7 \%$ (34) che dichiara di sentirsi più sicuro. Questi risultati sembrano dipendere in una certa misura dall'aver subito un reato: emerge infatti come la percentuale di chi ha risposto "più sicuro" sia più esigua $(28,2 \%)$ per coloro che sono stati vittime rispetto a coloro che non lo sono stati $(44,2 \%)$.

\section{Riflessioni conclusive.}

Confrontando i dati emersi dal questionario con quelli ottenuti dall'analisi di secondo livello emerge un dato inaspettato: nonostante i suoi 10 anni di attività, solo il 38,4\% (83) degli intervistati dichiara di essere a conoscenza del Centro e pochi di loro vi si sono rivolti per ricevere aiuto. Un dato questo di difficile interpretazione: una possibile lettura è che la funzione della struttura non sia ancora sufficientemente pubblicizzata o forse, ma è solo un'ipotesi, che gli altri enti locali presenti sul territorio non informino abbastanza i cittadini circa il servizio offerto.

Inoltre, ancorché il 21,3\% (46) di intervistati affermi che la presenza del Centro sul territorio non incida sulla propria percezione di sicurezza, si ritiene tuttavia che la percentuale del 15,7\% (34) di soggetti che si sentono più sicuri sia da ritenersi un dato significativo e confortante per un ulteriore sviluppo e potenziamento di questi centri.

Si vuole ora richiamare l'attenzione su quest'ultimo gruppo di persone: la maggior percezione di sicurezza legata alla presenza del Centro è correlata al non aver subito episodi vittimizzanti. Analogamente, la percezione di utilità dei centri di supporto è leggermente più elevata $(70,2 \%)$ per chi non è mai stato vittima rispetto a chi invece ha subito un reato $(66,3 \%)$.

Dall'analisi secondaria dei dati di accesso emerge come, nonostante la costante presenza nel tempo di problematiche legate a truffe e violenze domestiche, la stragrande maggioranza, pari al $61,7 \%$, dei casi trattati riguardi questioni di consumo e prestazioni, seguiti da disagi familiari e sociali $(28,9 \%)$. Questo dato è indice del fatto che le persone in difficoltà preferiscono avvalersi 
del Centro come soggetto erogatore di servizi di tipo economico-assistenziale, piuttosto che come interlocutore nella risoluzione di fatti-reato da loro patiti. Questo potrebbe essere dovuto ad una scarsa conoscenza di queste strutture e del servizio che offrono, oltre a un diffuso atteggiamento di scetticismo e diffidenza verso ciò che è nuovo e alternativo ai consueti percorsi.

A sostegno di questa interpretazione, che rimane solo una delle possibili, si riporta il fatto che nonostante la maggior parte $(68,5 \%)$ degli intervistati ritenga utile l'attività svolta dai centri di supporto alle vittime, queste strutture rimangono fra le ultime scelte da coloro che abbiano necessità di un aiuto. Infatti, indipendentemente da eventuali episodi di vittimizzazione subiti, gli stessi intervistati preferirebbero affidarsi in prima battuta a forze dell'ordine o avvocati piuttosto che a un centro di supporto.

Alla luce di tali problematicità e di quanto si è avuto modo di osservare sul campo, occorre delineare alcune necessità operative, relative al Centro di supporto alle vittime di reato e calamità di Casalecchio di Reno, ma estendibili anche ad altri centri di victim support affinché possano potenziare le proprie attività: innanzitutto si ritiene opportuno creare una solida rete di relazioni con le istituzioni presenti sul territorio. Dalla seppur breve esperienza effettuata presso il Centro si è riscontrato, per quanto concerne il piano relazionale, un buon grado di cooperazione fra volontari, ma si rileva invece qualche problematicità in ordine ai rapporti con Ausl e servizi sociali, quelle istituzioni esterne con le quali la sinergia dovrebbe essere più forte e che maggiormente dovrebbero collaborare e cooperare con il Centro. Si sottolineano infatti le difficoltà dell'operatore di un centro di supporto alle vittime proprio nella sua funzione di mediatore fra queste ultime e le istituzioni, oltre che nella concreta attività di aiuto, quando non supportato dalle istituzioni stesse. Seppure la presenza di un centro di supporto dovrebbe essere intesa quale valore aggiunto all'interno di una data realtà territoriale, in virtù della sua funzione di interlocutore preferenziale tra utenza bisognosa e istituzioni coinvolte nei singoli casi, è emerso come non di rado siano gli stessi operatori istituzionali a non tenere nella giusta considerazione l'operato dei volontari, inficiando dunque in alcuni casi i piccoli traguardi raggiunti nei confronti dell'utente stesso. Risulta quindi di fondamentale importanza la creazione di una rete stabile di scambi e di relazioni fra un centro di victim support e gli altri attori istituzionali presenti sul territorio, che dovrebbero operare di concerto a Forze dell'Ordine e servizi sociosanitari. L'unione fra questi attori è poi imprescindibile per un servizio di assistenza locale che voglia essere coordinato, riconosciuto e finanziato a livello nazionale.

In secondo luogo, è auspicabile una maggiore formazione: solide competenze sociocriminologiche, vittimologiche e psicologiche sono propedeutiche e funzionali alla comprensione e alla gestione di tutte le attività ordinarie che gli operatori di un centro di supporto alle vittime si trovano a dover gestire quotidianamente, a partire dalla prima e delicata fase di accoglienza dell'utente che non può essere affrontata a prescindere da una completa preparazione teorica. 
L'acquisizione di tali competenze, che certamente dovranno essere integrate con la prassi e con l'esperienza sul campo, è imprescindibile per la particolarità e la delicatezza dei casi con cui gli operatori, professionisti, sono chiamati a confrontarsi e a gestire. L'attività, inoltre, non può essere svolta esclusivamente da personale volontario: questo aspetto, intimamente legato al precedente, si riferisce al fatto che il lavoro deve essere svolto da personale qualificato, ognuno con una propria competenza specifica, scongiurando altresì il rischio di accentrare i compiti su un unico soggetto.

Infine, è di estrema importanza incrementare la promozione e l'informazione del servizio offerto dal Centro, chiedendo anche la collaborazione dei servizi pubblici comunali e non comunali (Carabinieri, Prefettura, Azienda Sanitaria Locale) oltre che dell'associazionismo territoriale.

I dati raccolti ed elaborati hanno apparentemente portato a confutare in via generale l'ipotesi iniziale per cui un centro di supporto alle vittime possa essere considerato come soggetto in grado di incrementare il sentimento di sicurezza degli attori sociali.

Si deve però considerare come tali dati siano riferibili esclusivamente al circoscritto territorio di Casalecchio di Reno e riguardino questo specifico Centro che presenta una particolarità nella sua storia e nella sua costituzione. Gli stessi dati poi non possono comunque essere considerati come statisticamente rappresentativi in quanto, a causa delle limitate risorse disponibili, non è stato possibile effettuare un vero e proprio campionamento statistico. La ricerca deve però essere letta e considerata come il risultato di un approccio metodologico ragionato agli strumenti di ricerca qualitativa e quantitativa propri delle scienze sociali.

Nella convinzione che il limitato ricorso a questi centri sia dovuto a una scarsa conoscenza delle attività di sostegno alle vittime, al termine di tale studio si ritiene che, se debitamente strutturati, organizzati e supportati a livello istituzionale, questi abbiano una grande potenzialità nell'offrire un maggior senso di sicurezza e nel ridurre la vulnerabilità diffusa tanto nelle vittime quanto nei soggetti più deboli (17). L'istituzione di centri di supporto alle vittime di reato rappresenta un primo passo per combattere l'isolamento, l'insicurezza, la non collaborazione e quel sentimento di sfiducia oggi così diffusi. In questo senso analoghe strutture di assistenza alle vittime possono verosimilmente rientrare all'interno di più ampie politiche di prevenzione sociale-terziaria che si occupano "[...] degli individui già condannati e delle vittime del crimine favorendo il reinserimento di tali soggetti nei propri ambienti di vita" (18), dal momento in cui pongono in essere interventi orientati al miglioramento della qualità vita nel contesto territoriale che possono essere efficacemente affiancati ad altre strategie preventive e repressive. I centri di supporto adottano infatti politiche di intervento volte a ripristinare il perduto senso di sé e a reintegrare il senso di responsabilità nell'individuo. Dalla convinzione che sia necessario ed imprescindibile affrontare la "questione sicurezza" a partire dal ripristino della coesione sociale, rilevando i bisogni e le vulnerabilità del cittadino in uno specifico territorio in termini di sicurezza, si crede che questa funzione potrebbe verosimilmente essere svolta dai centri di 
supporto alle vittime. Pensando alla società come a uno spazio relazionale, entro cui la polizia di prossimità è chiamata ad affiancare a tecniche e metodi già consolidati nuove politiche e competenze di mediazione, di dialogo e di ascolto per avvicinarsi ai cittadini e conoscerne in modo approfondito le esigenze, i centri di supporto alle vittime di reato, agendo a fianco di queste ultime, potrebbero svolgere proprio la funzione di informare le forze di polizia e le amministrazioni comunali circa le problematiche maggiormente sentite dai cittadini.

Alla luce di queste considerazioni, i centri di supporto sono da considerarsi soggetti utili al fine di incrementare il senso di sicurezza percepita: è stato infatti rilevato che "adempiendo a questa missione di prevenzione della vittimizzazione, i servizi alle vittime potranno essere in grado di portare un significativo contributo alla creazione ed al mantenimento di comunità più sicure" $(\underline{19})$.

\section{Note.}

(1) - Il 6 dicembre del 1990 un aereo militare in avaria, abbandonato dal pilota, si schianta contro un'aula della succursale dell'istituto tecnico commerciale Salvemini di Casalecchio di Reno, provocando la morte di 12 ragazzi e invalidità permanenti a 72 persone fra studenti, corpo docente e personale ausiliario. Per una disamina completa sulle vicende dell'incidente aereo occorso all'istituto si rimanda, inter alia a: Roffi G. (a cura di), Centro per le vittime di reato e calamità. Dalla strage del Salvemini un modello consolidato di solidarietà, Associazione Vittime del Salvemini 6 Dicembre 1990, Bologna, 2010; Associazione Vittime del Salvemini, Quindicesimo anniversario. Il progetto diventa realtà, un libro per ricordare la strage dell'istituto Salvemini, Seconda Ed., Comune di Casalecchio di Reno, 2005.

(2) - A riguardo si vedano, inter alia, Balloni A., Viano E., VI Congresso mondiale di vittimologia. Atti della giornata bolognese, Clueb, Bologna, 1989; Sette R., "Vittime e operatori del controllo sociale", in Bisi R. (a cura di), Vittimologia. Dinamiche relazionali tra vittimizzazione e mediazione, Franco Angeli, Milano, 2004.

(3) - A riguardo, si precisa che tali dati sono stati acquisiti dagli operatori e che sono pertanto stati utilizzati i medesimi indici di riferimento. In letteratura non è presente una definizione univoca di analisi secondaria, alcuni autori indicano con tale accezione una ricerca condotta su dati di inchiesta campionaria già precedentemente raccolti e disponibili nella forma della matrice dati originale, altri invece ne estendono la portata a tutte quelle elaborazioni statistiche di dati non direttamente rilevati dal ricercatore ma acquisiti da altre fonti ufficiali. In merito si veda ex multis Corbetta P., Metodologia e tecniche della ricerca sociale, op. cit., p. 222.

(4) - Corbetta P., Metodologia e tecniche della ricerca sociale, il Mulino, Bologna, 2014 pag. 367.

(5) - Sennett R., Insieme. Rituali, piaceri, politiche della collaborazione, Feltrinelli, Milano, 2012, p. 199.

(6) - Sette R., "Società sicure e mutamento sociale: possibili sfide per il futuro", in Rivista di Criminologia, Vittimologia e Sicurezza, Vol. VIII, N. 1, GennaioAprile 2014, pag. 110.

(7) - Bisi R., "Vittimizzazione: l'imprevedibilità di un percorso e la necessità di uno studio", in Balloni A., Bisi R., Costantino S., (a cura di), Legalità $e$ comunicazione. Una sfida ai processi di vittimizzazione, FrancoAngeli, Milano, 2008, pp. 36-37.

(8) - I gruppi Facebook cui si è fatto riferimento sono "Sei di Casalecchio se..."; "Se sei di Casalecchio e senza pregiudizi se..."; "Commercianti E Cittadini Casalecchio di Reno"; "forum Casalecchio...". Non si è invece ottenuta autorizzazione da parte degli amministratori dei gruppi "Casalecchio insieme Pro Loco" e "Il Blogos", entrambi gestiti da personale afferente all'amministrazione comunale.

(9) - Per la costruzione del questionario si sono consultati Pellicciari G., "Il questionario e l'intervista" e "Come si fa un questionario" in Guidicini P., Nuovo manuale della ricerca sociologica, Franco Angeli, Milano, 1987 oltre a Cremonini F., "Rilevazione diretta di informazioni: esemplificazione di un percorso di indagine tramite questionario", in Cremonini F. (a cura di) Strumenti e tecniche per l'indagine criminologica. Una introduqione, Franco Angeli, Milano, 2002; Corbetta P., Metodologia e tecniche della ricerca sociale, op. cit.; Babbie E., Ricerca sociale, Apogeo, Milano, 2010.

(10) - Per la distinzione in ambiti e assi tematici si rimanda, inter alia, a Cremonini F., "Rilevazione diretta di informazioni: esemplificazione di un percorso di indagine tramite questionario", in Cremonini F. (a cura di), op. cit., pp. 71 e ss.

(11) - Proprio nei giorni in cui è stato somministrato il questionario (mese di novembre 2014), infatti, i commercianti di Casalecchio di Reno hanno presentato una petizione al Comune lamentando degrado e controlli insufficienti da parte delle autorità.

(12) - Forlivesi A., Criminalità, rischio e sicurezza, Clueb, Bologna, 2004, pag.87.

(13) - Bisi R., "Vittimizzazione: l'imprevedibilità di un percorso e la necessità di uno studio" in Balloni A., Bisi R., Costantino S., Legalità e comunicazione. Una sfida ai processi di vittimizzazione, op. cit., pag. 31.

(14) - Il riferimento è qui alla ricerca "Processi di vittimizzazione e programmi di sostegno alle vittime: 
due realtà a confronto, l'Emilia-Romagna e la Sicilia", condotta dall'unità operativa dell'Università di Bologna. Per approfondimenti si veda Sette R., "Processi di vittimizzazione fra realtà e stereotipi, in Legalità e comunicazione. Una sfida ai processi di vittimizzazione", in Balloni A., Bisi R., Costantino S., (a cura di), op. cit., pag. 67.

(15) - Sicurella S., Vittime e istituzioni locali. Quale dialogo?, Clueb, Bologna, 2010, pag. 83.

(16) - Sicurella S., Ibidem, pag. 85.

(17) - A tal proposito si veda C. Zarafonitou, "Victims' insecurity and criminal policy: The role of victim's support services", in Rivista di Criminologia, Vittimologia e Sicurezza, anno VIII, n. 1, GennaioAprile 2014.

(18) - Sette R, Controllo sociale e prevenzione. Un approccio criminologico, Clueb, Bologna, 2008, pag. 109.

(19) - Sette R., "Vittime e operatori del controllo sociale", in Bisi R. (a cura di), Vittimologia. Dinamiche relazionali tra vittimizzazione e mediazione, op. cit., pag. 50 .

\section{Bibliografia.}

- Babbie E., Ricerca sociale, Apogeo, Milano, 2010.

- Balloni A. (a cura di), Vittime Crimine Difesa sociale, Clueb, Bologna, 1989.

- Balloni A., "La vittima del reato, questa dimenticata", in Atti tavola rotonda della conferenza annuale della ricerca (5 dicembre 2000), Accademia Nazionale dei Lincei, Roma, 2001.

- Balloni A. (a cura di), Il vigile di quartiere a Milano. Percorsi formativi e operativi: un approccio criminologico, FrancoAngeli, Milano, 2003.

- Balloni A. (a cura di), Cittadinanza responsabile e tutela della vittima, Clueb, Bologna, 2006.

- Balloni A., Bisi R. (a cura di), "Processi di vittimizzazione e reti di sostegno alle vittime", in Salute e Società, n.1, Franco Angeli, Milano, 2008.

- Balloni A., Bisi R., Costantino S. (a cura di), Legalità e comunicazrione. Una sfida ai processi di vittimizzazione, FrancoAngeli, Milano, 2008.

- Balloni A., Bisi R., Sette R. (a cura di), Vittime e Vittimologia. Percorsi di studio e di ricerca, Minerva, Bologna, 2012.

- Balloni A., Bisi R., Sette R., Manuale di Criminologia - I. Le teorie, Clueb, Bologna, 2013.

- Balloni A., Bisi R., Sette R., Manuale di Criminologia - II. Criminalità, controllo sicurezza, Clueb, Bologna, 2013.

- Balloni A., Viano E. (a cura di), IV Congresso mondiale di vittimologia: atti della giornata bolognese, Clueb, Bologna, 1989.
- Bisi R. (a cura di), Vittimologia. Dinamiche relazionali tra vittimizzazione e mediazione, FrancoAngeli, Milano, 2004.

- Bisi R., Faccioli P. (a cura di), Con gli occhi della vittima. Approccio interdisciplinare alla vittimologia, FrancoAngeli, Milano, 1996.

- Castel R., L'insicurezza sociale. Che significa essere protetti?, Einaudi, Torino, 2004.

- Codini G. (a cura di), La vittimologia e le vittime fragili : la situazione in Europa e $i$ servizi di supporto, FrancoAngeli, Milano, 2010.

- Corbetta P., Metodologia e tecniche della ricerca sociale, Seconda edizione, il Mulino, Bologna, 2014.

- Cremonini F. (a cura di), Strumenti e tecniche per l'indagine criminologica, FrancoAngeli, Milano, 2002.

- Dieu F., "La victime, acteur de la sécurité?", in Rivista di Criminologia, Vittimologia e Sicurezza, Vol. VI, N. 2, Maggio-Agosto 2012.

- Ferrarotti F., Storia e storie di vita, Laterza, Bari, 1997.

- Forlivesi A., Criminalità, rischio e sicurezza. Analisi e prospettive, Clueb, Bologna, 2004.

- Guidicini P., Pieretti G., I volti della povertà urbana, FrancoAngeli, Milano, 1988.

- Guidicini P., Nuovo manuale della ricerca sociologica, FrancoAngeli, Milano, 1987.

- Guidicini P., Questionari, Interviste, Storie di vita: come costruire gli strumenti, raccogliere le informazioni ed elaborare $i$ dati, Franco Angeli, Milano, 1995.

- Gulotta G., Vagaggini M. (a cura di), Dalla parte della vittima, Giuffrè, Milano, 1981.

- Marotta G., Teorie criminologiche. Da Beccaria al postmoderno, LED, Milano, 2004.

- Marradi A., Concetti e metodo per la ricerca sociale, La Giuntina, Firenze, 1997.

- Marradi A., L'analisi monovariata, FrancoAngeli, Milano, 1995.

- Marradi A., Linee guida per l'analisi bivariata dei dati nelle scienze sociali, FrancoAngeli, Milano, 1997.

- Prandini R., Le radici fiduciarie del legame sociale, FrancoAngeli, Milano, 1998.

- Roffi G. (a cura di), Centro per le vittime di reato e calamità. Dalla strage del Salvemini un modello consolidato di solidarietà, Associazione Vittime del Salvemini 6 Dicembre 1990, Bologna, 2010.

- Sette R., Controllo sociale e prevenzione. Un approccio criminologico, Clueb, Bologna, 2008. 
- Sette R., Criminologia e Vittimologia. Metodologie e strategie operative, Minerva, Bologna, 2011.

- Sette R., "Società sicure e mutamento sociale: possibili sfide per il futuro", in Rivista di Criminologia, Vittimologia e Sicurezza, Vol. VIII, N. 1, Gennaio-Aprile 2014.

- Sicurella S., "Victims of crime and society: student's opinion", in Rivista di Criminologia, Vittimologia e Sicurezza, Vol. II, N. 3, Settembre-Dicembre 2008.

- Sicurella S., Vittime e istiturioni locali. Quale dialogo?, Clueb, Bologna, 2010.

- Sicurella S., La vulnerabilità sociale nei contesti urbani, Clueb, Bologna, 2012.

- Zarafonitou C., "Victims' insecurity and criminal policy: The role of victim's support services", in Rivista di Criminologia, Vittimologia e Sicurez:a, Vol. VIII, N. 1, Gennaio-Aprile 2014. 